J Int Neuropsychol Soc. 2021 November ; 27(10): 1037-1047. doi:10.1017/S1355617721000096.

\title{
Everyday Executive Function and Self-Awareness in Agenesis of the Corpus Callosum
}

\author{
Ryan W. Mangum ${ }^{1}$, Justin S. Miller ${ }^{1}$, Warren S. Brown ${ }^{1,2}$, Anne A.T. Nolty ${ }^{1}$, Lynn K. \\ Paul ${ }^{1,2,3,{ }^{*}}$ \\ ${ }^{1}$ Fuller Graduate School of Psychology, Travis Research Institute, Pasadena, CA, USA \\ 2International Research Consortium for the Corpus Callosum and Cerebral Connectivity (IRC5), \\ Pasadena, CA, USA \\ ${ }^{3}$ California Institute of Technology, Division of Humanities and Social Sciences, Pasadena, CA, \\ USA
}

\section{Abstract}

Objective: Agenesis of the corpus callosum $(\mathrm{AgCC})$ is associated with a range of cognitive deficits, including mild to moderate problems in higher order executive functions evident in neuropsychological assessments. Previous research has also suggested a lack of self-awareness in persons with AgCC.

Method: We investigated daily executive functioning and self-awareness in 36 individuals with $\mathrm{AgCC}$ by analyzing self-ratings on the Behavior Rating Inventory of Executive Function-Adult Version (BRIEF-A), as well as ratings on the same instrument from close relatives. Discrepancies between self- and informant-ratings were compared to the normative sample and exploratory analyses examined possible moderating effects of participant and informant characteristics.

Results: Significant deficiencies were found in the Behavioral Regulation and Metacognitive indices for both the self and informant results, with elevated frequency of metacognition scores in the borderline to clinical range. Informants also endorsed elevated frequency of borderline to clinically significant behavioral regulation scores. The proportion of $\mathrm{AgCC}$ participants whose self-ratings indicated less metacognitive impairment than informant-ratings was greater than in the normative sample. Self-ratings of behavioral regulation impairment decreased with age and informant-ratings of metacognition were higher in males than females.

Conclusions: These findings provide evidence that individuals with $\mathrm{AgCC}$ experience mild to moderate executive functioning problems in everyday behavior which are observed by others. Results also suggest a lack of self-understanding or insight into the severity of these problems in the individuals with $\mathrm{AgCC}$, particularly with respect to their metacognitive functioning.

\footnotetext{
*Correspondence and reprint requests to: Lynn K. Paul, Baxter MC 228-77, California Institute of Technology, 1200 E California Blvd, Pasadena, CA 91125, USA. lkpaul@hss.caltech.edu.

CONFLICTS OF INTEREST

The authors have no conflicts of interest.
} 


\section{Keywords}

Behavioral regulation; cognitive flexibility; executive function; metacognition; self-awareness; interhemispheric commissure; BRIEF-A

Agenesis of the corpus callosum ( $\mathrm{AgCC})$ is a congenital anomaly involving complete or partial absence of the band of white matter fibers connecting brain hemispheres (Jinkins, Whittemore, \& Bradley, 1989). AgCC is associated with various mild to moderate cognitive deficits, including deficits in executive functioning (e.g., Brown, Anderson, Symington, \& Paul, 2012; Brown \& Paul, 2000, 2019; Marco et al., 2012). Self-monitoring is an aspect of executive functioning that has not been studied in $\mathrm{AgCC}$, but evidence from self-report personality measures suggests this is an area of weakness (Brown \& Paul, 2000; Kaplan, Brown, Adolphs \& Paul, 2012; Longino, 2011).

The aim of this investigation was to study everyday executive functioning in individuals with $\mathrm{AgCC}$, and the accuracy of their self-perception regarding this area of functioning. This was achieved by examining self- and informant-ratings of daily executive functioning using the Behavioral Rating Inventory of Executive Function-Adult version (BRIEF-A; Roth, Isquith, \& Gioia, 2005).

\section{Agenesis of the Corpus Callosum}

AgCC occurs in an estimated 1 of every 4000 live births (Glass, Shaw, Ma, \& Sherr, 2008), and involves complete or partial absence of the corpus callosum. While the etiology of $\mathrm{AgCC}$ is not fully understood, it is thought to arise from a combination of both genetic and environmental factors (Paul et al., 2007). Individuals with AgCC may develop and retain commissures other than the corpus callosum (such as the anterior commissure), and some degree of interhemispheric transfer may occur through these alternative commissures (Barr \& Corballis, 2002; Brown, Jeeves, Dietrich, \& Burnison, 1999; Brown, Thrasher, \& Paul, 2001; Fischer, Ryan, \& Dobyns, 1992). The functioning of individuals with complete $\mathrm{AgCC}$ is therefore not the same as that of individuals with a commissurotomy ("split-brain"; Sperry, 1974). Nonetheless, interhemispheric transfer of information is less efficient in non-callosal pathways when compared to transfer through the corpus callosum (Brown et al., 1999; Brown et al., 2001).

There is a significant sub-population of individuals with $\mathrm{AgCC}$ who are without other brain abnormalities and possess a full-scale intelligence quotient within the normal range (i.e., FSIQ 280; Chiarello, 1980; Paul et al., 2007; Sauerwein \& Lassonde, 1994). This clinical presentation, called primary AgCC (Brown \& Paul, 2019), provides opportunities for the focused study of the neuropsychological impact of complete or partial absence of the corpus callosum.

\section{Cognitive Profile of AgCC}

A growing body of research outlines the typical pattern of mild to moderate cognitive and psychosocial deficits in Primary AgCC (reviewed by Brown \& Paul, 2019; Siffredi, 
Anderson, Leventer \& Spencer-Smith, 2013). Brown and Paul (2019) have argued that the core deficiencies in individuals with Primary AgCC involve (a) interhemispheric transfer of complex sensory information and learning (Brown et al., 1999; Imamura, Yamadori, Shiga, Sahara, \& Abiko, 1994; Jeeves, 1979; Karnath, Schumacher, \& Wallesch, 1991; Sauerwein \& Lassonde, 1983), (b) cognitive processing speed (Erickson, Young, Paul, \& Brown, 2013; Marco et al., 2012), and (c) complex novel problem-solving (e.g., Brown et al., 2012; Schieffer, Paul, \& Brown, 2000; Fischer et al., 1992; Gott \& Saul, 1978; Sauerwein \& Lassonde, 1994; Smith \& Rourke, 1995; Solursh, Margulies, Ashem, \& Stasiak, 1965).

More specifically, across many studies, individuals with AgCC exhibit deficits in a variety of cognitive and psychosocial domains, including: learning and recall of verbal and visual information (Erickson, Paul, \& Brown, 2014; Paul, Erickson, Hartman, \& Brown, 2016), comprehension of second-order meanings of language and proverbs (Brown, Paul, Symington, \& Dietrich, 2005; Brown, Symington, VanLancker-Sidtis, Dietrich, \& Paul, 2005; Paul, VanLancker-Sidtis, Schieffer, \& Brown, 2003; Rehmel, Brown, \& Paul, 2016), recognition of cues to emotion in faces (Bridgman et al., 2014), interpreting sarcasm and understanding subtle aspects of social interactions (Symington, Paul, Symington, Ono, \& Brown, 2010), and imagining and inferring the mental, emotional, and social functioning of others (Renteria-Vazquez et al., in press; Turk, Brown, Symington, \& Paul, 2010; Young et al., 2019). While general intelligence is not deficient among individuals with Primary $\mathrm{AgCC}$, they appear to struggle with tasks demanding fluid intelligence compared to those involving crystallized or static intelligence (Brown \& Paul, 2000, 2019).

\section{$\mathrm{AgCC}$ and Executive Functions}

Studies of AgCC using clinical assessment tools have found mild executive function deficits, particularly manifest in response inhibition and switching (Marco et al, 2012), sustained attention and vigilance (Brown, Panos \& Paul, 2020), encoding in list-learning (Erickson et al., 2014; Paul et al., 2016), and strategic understanding and use of interpersonal emotions (Anderson, Paul, \& Brown, 2017). As described by Brown and Paul (2019), more pronounced executive function deficiencies are evident in problem-solving. Evidence of difficulty with complex, novel problems can be found in several domains, including decision-making and social functioning. Impairments in decision-making have been demonstrated on categorization tasks (Schieffer, 1999) and the Iowa Gambling Task (Bechara, Damásio, Damásio, \& Anderson, 1994; Brown et al., 2012). Results from the Iowa Gambling Task suggest that in AgCC, decision-making is specifically hindered by difficulty establishing a beneficial strategy (Brown et al., 2012). In the social domain, individuals with $\mathrm{AgCC}$ had difficulty inferring the mental states of the people (i.e., theory of mind) shown in videos depicting complex social interactions (Symington et al., 2010) or inferring social intention from the interpersonal-like interactions suggested by animated triangles (Renteria-Vazquez et al., in press). Individuals with complete $\mathrm{AgCC}$ also demonstrated impairments in awareness of the consequences of action choices (Young et al., 2019), as well as in logical storytelling, social insight, and appreciation of common story content when responding to stimuli from the Thematic Apperception Test (TAT; Paul, Schieffer, \& Brown, 2004; Turk et al., 2010). 
Finally, AgCC appears to interfere with higher order language skills, such as understanding the second-order meanings of non-literal language expressions found in proverbs (Rehmel et al., 2016), idioms (Paul et al., 2003), and humor (Brown, Paul, et al., 2005). Recognition of second-order meanings requires a shift in the semantic field of interpretation. As Rehmel and colleagues (2016) pointed out, difficulties with understanding second-order meanings and complex linguistic forms (such as proverbs) may account for the broader social deficiencies that individuals with AgCC experience in daily life. Similar problems are also evident in diminished ability to imagine the consequences of alternative behavioral decisions (Young et al., 2019).

Poor executive functioning may impact multiple domains of everyday life. By early childhood, parents have reported marked behavioral problems in their children with $\mathrm{AgCC}$ in the areas of attention, socialization, and thought processes (Badaruddin et al., 2007). Although some adults with Primary AgCC may function relatively well in highly routinized social interactions, in less familiar or more complicated situations they may exhibit symptoms consistent with autism spectrum disorder (Paul, Corsello, Kennedy, \& Adolphs, 2014). This suggests that it is the highly complex and novel domains of problemsolving that pose the greatest problems for individuals with AgCC. Brown and Paul (2000, 2019) hypothesized that AgCC leads to diminished ability for higher cognitive information processing, but that the manifestation of this diminished ability may not show up in simpler, over-learned, or rote tasks.

\section{Awareness of Deficits in AgCC}

On self-report measures, adults with AgCC reveal a pattern of naïve self-understanding and inaccurate perceptions regarding their cognitive and social challenges that suggest limited self-awareness. For example in adults with AgCC, profiles generated from a selfreport personality inventory (Minnesota Multiphasic Personality Inventory-Second Edition, MMPI-2 and MMPI-2-RF, Butcher et al., 2001) were characterized by a lack of selfawareness, significant psychological naïveté, unsophisticated defenses, and deficient selfunderstanding (Brown \& Paul, 2000; Kaplan et al., 2012; Longino, 2011). In these studies, elevations were found on the L-scale which were attributed to deficient social insight and poor personal awareness. Longino (2011) further highlighted the parallels between MMPI-2 profiles among the AgCC and autism spectrum disorder populations, both of which manifest "limited insight and a lack of awareness of how one's behaviors might affect others" (p. 19).

Limited self-awareness in AgCC was also evident in a screening measure for cognitive and behavioral traits of autism. On this measure, adults with AgCC reported lower incidence of autism traits on self-ratings (Lau et al., 2013) than were reported by their parents, who were presumed to provide more objective and accurate ratings. Taken together, these studies suggest a pattern of poor self-awareness in AgCC, specifically with regard to social and interpersonal skills, but little is known about their self-awareness regarding other areas of potential weakness such as executive functioning in daily activities.

J Int Neuropsychol Soc. Author manuscript; available in PMC 2022 January 10. 


\section{Rationale and Hypotheses}

While results from neuropsychological assessment instruments have indicated that individuals with $\mathrm{AgCC}$ have executive function difficulties, effective treatment planning requires insight about how these deficits manifest themselves in everyday behaviors and how these problems in everyday behavior are understood by individuals with AgCC. Thus, this study used the Behavior Rating Inventory of Executive Function-Adult Version (BRIEF-A) to ascertain and compare self- and informant-ratings of daily executive functioning in adults with AgCC. To identify perceived difficulty in everyday behavior, standardized BRIEF-A scores from each rater were statistically compared to the mean of a normal distribution.

Additionally, participant accuracy of self-perception (i.e., self-awareness) was examined using the discrepancy between self- and informant-ratings for each participant, with the presumption that informant-ratings provide a more objective index of functioning. This approach is consistent with studies of individuals with autism spectrum disorder (Verhoeven et al., 2012) and traumatic brain injury (Bivona et al., 2008) which reported impaired self-awareness based on discrepancies between parent-reports and self-reports. Furthermore, a study of self-awareness in individuals with traumatic brain injury found greater parent/selfreport discrepancies on BRIEF ratings of metacognition than behavioral regulation (Wilson Donders, \& Nguyen, 2011), suggesting that the BRIEF Metacognition Index (MI) may be a particularly sensitive index of brain-based impairment in deficit awareness.

We hypothesized that deficiencies would be evident particularly on the MI since the behaviors measured by this index involve complex cognitive abilities, such as problemsolving, planning, and organizing (Roth et al., 2005; Strauss, Sherman, \& Spreen, 2006). In addition, we hypothesized that participants with $\mathrm{AgCC}$ would endorse less difficulty with executive function than their informants, indicating mild deficiencies in self-awareness.

\section{METHOD}

\section{Participants}

Thirty-six participants with $\mathrm{AgCC}$ ( 18 females, 18 males) between the ages of 18 and 72 years $(M=33.72, S D=13.79)$, with Full-Scale Intelligence Quotient (FSIQ) in the normal range ( $M=100.14, S D=12.41$, range 78-129) completed questionnaires measuring executive behaviors. The sample was predominately Caucasian (80\%). Regarding education, $22 \%$ graduated high school, $39 \%$ completed some college, $31 \%$ completed college, and $8 \%$ completed an advanced degree. Criteria for inclusion in this study were: complete or partial AgCC confirmed by neuroimaging, at least 18 years of age, normal-range FSIQ (i.e., $>75$ ), and at least a seventh-grade reading level. From this sample, $\mathrm{AgCC}$ diagnosis was confirmed in 22 participants through MRI review (16 complete, 6 partial; 13 isolated, 9 $\mathrm{AgCC}$ plus other mild brain dysmorphology). For 14 participants, inclusion in the $\mathrm{AgCC}$ group was based on clinical diagnosis via MRI, with details from the MRI report provided by the informant ( 6 with complete, 4 with partial, 2 with dysgenesis, and 2 unknown; 9 isolated, $3 \mathrm{AgCC}$ plus other neuropathology, and 2 unknown). Ten participants were also included in the Marco et al. (2012) study. Participants with AgCC were recruited 
through announcements distributed by the National Organization for Disorders of the Corpus Callosum and participant-initiated contact with the authors.

Each participant had one informant who also completed the BRIEF-A. Informants were selected by AgCC participants and were adults close enough to the participant to have observed their daily behavior in a variety of settings and over a sufficient amount of time. Informants included parents (78\%), siblings (14\%), and spouse or partner (8\%). Participants rated by siblings or partners were evenly distributed by gender ( 4 males, 4 females).

\section{Procedure}

Participants with AgCC completed the BRIEF-A and identified an individual familiar with their daily behavior who completed the BRIEF-A informant version. Informants were also asked to provide background history (e.g., medical, developmental, and educational) on behalf of the AgCC participant. Before taking the BRIEF-A, participants with AgCC had to successfully complete the Wechsler Individual Achievement Test, Second Edition (WIAT-II; Wechsler, 2005) Reading Comprehension item that demarcates seventh-grade reading level (as required for the BRIEF-A, Roth et al., 2005). In 14 participants, FSIQ was acquired previously in the ongoing AgCC study at Travis Research Institute using Wechsler Adult Intelligence Scale, Third Edition (WAIS-III; Wechsler, 1997, $n=13$ ) or the Wechsler Intelligence Scale for Children, Fourth Edition (Wechsler, 2003, $n=1$, at age 15). FSIQ was estimated in the remaining 22 participants by administration of the Wechsler Test of Adult Reading (WTAR; Wechsler, 2001), which correlates strongly with WAIS-III FSIQ in the age-range of our sample (.70-.80, Wechsler, 2001).

Self- and informant-reports were collected using a secure online questionnaire in Qualtrics. The reading comprehension screen (i.e., WIAT-II subtest) was integrated into the online questionnaire and administered prior to the BRIEF-A. Licensing keys were obtained for all test and questionnaire items. All individuals with $\mathrm{AgCC}$ and their informants provided consent for participation via online consent forms. The Institutional Review Board of the Travis Research Institute at Fuller Graduate School of Psychology approved all procedures for this study.

\section{MEASURES}

\section{Behavior Rating Inventory of Executive Function-Adult Version (BRIEF-A)}

The BRIEF-A (Roth et al., 2005) is a standardized questionnaire used to assess performance of self-regulatory behaviors in everyday life in adults aged 18-90. Both the self- and informant-forms of the BRIEF-A contain 75 statements on which respondents rate the level of functioning with a 3-point Likert scale of Never, Sometimes, or Often. Responses are summed into 9 clinical scales and 3 composite indices that are norm-referenced by age and converted to $T$ scores $(M=50, S D=10)$, with elevations indicating greater impairment. $T$ score norms for the BRIEF-A are based on data collected from 1,136 self-reports and 1,200 informant-reports as detailed in the test manual (Roth et al., 2005, pp. 52-53).

The two composite indices, Behavioral Regulation Index (BRI) and Metacognition Index (MI) are comprised of subscales as follows: BRI—Inhibit, Shift, Emotional Control, 
and Self-Monitor; MI—Initiate, Working Memory, Plan/Organize, Task Monitor, and Organization of Materials. The BRI represents the ability to maintain appropriate regulatory control over behavior and emotional responses, while the MI represents the ability to systematically solve problems via planning and organizing while sustaining processes in working memory systems. The Global Executive Composite (GEC), comprised of both BRI and MI, provides an overall summary rating of an individual's executive functioning. The BRIEF-A also incorporates 3 validity scales: the Negativity Scale sums items that are unusually negative, the Infrequency Scale sums atypical responses, and the Inconsistency Scale sums the number of times similar questions are answered in opposite directions. For both forms, internal consistency coefficients from the normative group, $r=.73-.96$, and test-retest reliability correlations for a subset of healthy adults, $r=.82-.96$, are adequate for all scales and subscales (Roth et al., 2005, p. 61).

\section{Data Analysis}

Statistical analyses were conducted in SPSS. Self- and informant-reports were analyzed separately using one-sample $t$ tests to compare $T$ scores with the normative mean (50) for each composite index. Significant findings were followed by post-hoc one-sample $t$ tests of subscales. Adjustment for multiple-comparisons was addressed with a modified Bonferroni correction of significance for comparison of the 2 composite indices, and Bonferroni corrections for exploratory analyses (for 4 BRI subtests and for 5 MI subtests). The number of participants with composite index scores at or above the threshold considered borderline clinically significant (i.e., one standard deviation above the normative mean; $T$ score 260 ) was compared to the number expected in a normal distribution, and significant findings (corrected for multiple comparisons) were followed by post-hoc examination of subtest scores. Fisher's Exact Test indicates that 13 of 36 individuals with $T$ scores greater or equal to 60 would constitute a statistically significant proportion $\left(X^{2}=4.74, p=.029\right)$ for a single measure, and 14 of 36 would be significant for two measures using Bonferroni correction $\left(X^{2}=5.79, p=.016\right)$.

To directly compare composite scale ratings of the same individual completed by two raters (self and informant), we calculated a discrepancy score for each participant (selfminus informant-rating). Using a Chi-square test (Campbell, 2007; Richardson, 2011; Altman, Machin, Bryant, \& Gardner, 2000), we compared the proportion of participants with discrepancy scores over 1 standard deviation (in each direction) to the proportion of participants in the mixed clinical/healthy normative sample with discrepancy scores in that range (Roth et al., 2005, p.65).

Effects of informant characteristics (informant-participant relationship, parent or other, and informant gender) and participant characteristics (age, gender, and FSIQ) on the primary dependent measures of the study (BRI and MI) were probed via stepwise regression with self-versus-informant discrepancy scores. Although use of age-adjusted $T$ scores controls for normal age-related variation, additional analysis of age effects was conducted because it is possible that age may have a greater impact in a clinical group, resulting in more typical age-adjusted scores at some ages and more atypical age-adjusted scores at other ages. 


\section{RESULTS}

\section{Validity Scales}

No subjects were excluded due to validity scale scores. On the Negativity scale, informantratings were significantly higher than self-ratings, $F(1,35)=16.59, p<.001, \eta p^{2}=.322$. Only one informant-rating approached significant elevation (raw score $=6$ ), but all index and subscale ratings for this participant fell within the distribution of the entire sample.

There was a trend toward significantly lower Infrequency scores from informants, $F(1,34)$ $=4.10, p=.051, \eta p^{2}=.108$. Infrequency was elevated for two informant-ratings and one self-rating (raw score $=3$ ), but these protocols were retained in our sample since all of their scores were quite low ( $T$ score range $=32-47$ ) and they would only reduce the possibility of finding elevations.

Informant- and self-ratings did not differ on Inconsistency scores, $F(1,35)=1.94, p=.173$, $\eta p^{2}=.053$. Inconsistency was elevated for one informant (raw score $=8$ ) and 2 participants (raw scores of 8 and 9). None of the index and subscale ratings for these participants were outliers relative to the total sample, so all were retained in the analyses.

\section{BRIEF-A Scores}

Detailed descriptive and $t$ test statistics are listed in Tables 1 and 2. Self-ratings of executive functioning were significantly elevated compared to test norms on both the BRI, $d=.94$, and MI, $d=.99$ (with Bonferroni correction). Post-hoc analyses revealed significant elevations on six subscales (Shift, Emotional Control, Initiate, Working Memory, Plan/Organize, and Task Monitor) with moderate to large effect sizes (BRI subscales $d>1.00$, MI subscales $d>$.74). A significant percentage of participants scored at or above the borderline-clinical threshold for MI, but not for BRI (with Bonferroni correction). Post-hoc comparisons of MI subscales revealed a significant percentage of participants with elevations on Initiate, Working Memory, Plan/Organize, and Task Monitor (see Table 1). In exploratory analysis of BRI subtests, 15 of 36 would be significant using Bonferroni correction, $X^{2}=6.92, p=.009$, and only Shift met that threshold $(n=21)$.

Informant-ratings were also significantly elevated compared to test norms on both BRI, $d=.90$, and MI, $d=1.36$. Informants endorsed elevations on the same subscales as the self-ratings (with the addition of Organization of Materials) and effect sizes were also moderate to large (BRI subscales $d>.77$, MI subscales $d>1.08$ ). Additionally, there was a trend toward significant elevation on the Self-Monitor subscale with a moderate effect size, $d=.67$. A significant percentage of participants had informant-ratings at or above the borderline-clinical threshold for both MI and BRI (with Bonferroni correction), as well as on all MI subscales and on Shift and Self-Monitor subtests of BRI (see Table 2).

\section{Self- Versus Informant-Ratings}

The $T$ score discrepancy between self- and informant-ratings was calculated for both index scores and all subscales. Table 3 presents the frequency of discrepancy scores of 1 standard deviation or greater in each direction. The proportion of $\mathrm{AgCC}$ participants whose self- 
ratings indicated less impairment than informant-ratings (discrepancy $\geq 1 S D$ ) was greater than in the normative sample for the metacognition index, $X^{2}=10.05, p=.0015$ (Bonferroni corrected for 2 index scores), as well as the initiate, $X^{2}=5.47, p=.0193$, planning, $X^{2}=$ $6.95, p=.0084$, and organization of materials, $X^{2}=6.98, p=.0082$, subscales. In contrast, frequency of $1 S D$ or greater discrepancies on the behavioral regulation index was similar in the $\mathrm{AgCC}$ and normative samples. However, in exploratory analyses, the proportion of $\mathrm{AgCC}$ participants whose self-ratings indicated less impairment than informant-ratings was greater than in the normative sample for the shift, $X^{2}=10.78, p=.001$, and self-monitor, $X^{2}$ $=8.27, p=.004$, subscales (Bonferroni corrected for 4 subscales).

\section{Exploration of Moderating Effects of Informant and Participant Characteristics}

Informant characteristics (relationship with participant, informant gender) and participant characteristics (FSIQ, age, gender) were entered into a stepwise regression with self-versusinformant discrepancy scores on BRI and MI. For BRI discrepancy scores, the regression model accounted for only $11 \%$ of the variance, $R^{2}=.142, F(1,34)=5.63, p=0.023$ and participant age was the only significant predictor, $\beta=.296, t=2.37, p=0.023,95 \% \mathrm{CI}$ $.042, .549$. Younger participants were more likely to endorse greater behavioral regulation impairment than their informants, while older participants were more likely to endorse less impairment than their informants. Post-hoc correlations suggest that age primarily influenced self-reported BRI scores, $r=-.303, p=.073$, and had minimal impact on informant reports, $r=.045, p=.794$.

For MI discrepancy scores, the regression model accounted for only $10 \%$ of the variance, $R^{2}$ $=.128, F(1,34)=5.01, p=0.32$ and participant gender was the only factor that predicted MI discrepancy, $\beta=-10.111, t=2.24, p=0.032,95 \%$ CI .931, 19.291. On average, informant-reported metacognition deficits were more discrepant from self-reports in males than females (males $M=7.94, S D=16.06$; females $M=-2.17, S D=10.46 ; t(34)=$ $-2.24, p=.032, d=.75)$. Self-reported MI did not differ in male and female participants, but informants reported greater metacognition deficits in males with $\mathrm{AgCC}$ than in females (males $M=64.28, S D=13.56$; females $M=54.78, S D=13.68 ; t(34)=-2.09, p=.044$, $d=.70)$. In fact for males, compared to a normal distribution informant-ratings indicated significant metacognition impairment overall, $t(17)=4.469, p<.001, d=1.05$, and on all subscales ( $d$ range .72 to 1.06 ), with a significant percentage of male participants at or above the borderline-clinical threshold, $X^{2}=6.41, p=.011$. In contrast, the only indication of impaired metacognition in females was on self-rating of working memory, $t(17)=3.035$, $p=.007, d=1.47$ (with Bonferroni correction for 5 subscales).

\section{DISCUSSION}

This study investigated difficulties in everyday executive function among adults with AgCC and characterized the accuracy of their self-perception regarding these abilities. Both self- and informant-reports confirmed that on average, adults with $\mathrm{AgCC}$ have difficulties with daily executive functioning, and a significant percentage of these individuals have borderline to clinically elevated scores in metacognition and set-shifting. In comparison to 
norms, informants reported larger deficits in metacognition and set-shifting than participants reported.

The proportion of $\mathrm{AgCC}$ participants whose self-ratings indicated less impairment than informant-ratings (discrepancy $\geq 1 S D$ ) was greater than in the normative sample for the metacognition index, as well as the initiate, plan, and organization of materials subscales. Although the frequency of large BRI discrepancy scores in AgCC did not differ from norms, the proportion of participants with $\geq 1 S D$ discrepancy between self and informant-ratings was greater than the normative sample for shift and self-monitor subscales.

Exploratory analyses suggest that discrepancies between self- and informant-ratings of behavioral regulation are associated with changes in self-awareness across the age range, while discrepancies in metacognition ratings are influenced by greater informant-ratings of deficits in males.

\section{BRIEF-A Ratings}

Individuals with $\mathrm{AgCC}$ endorsed deficits in both behavioral regulation and metacognition. Elevated subscale scores and large effect sizes suggest they see themselves as having problems with being flexible and making transitions, modulating and controlling emotions, and holding information in mind (i.e., poor working memory-that is, often losing track of what they are doing and being easily distracted). They also endorsed difficulty initiating, planning, and monitoring their own activities. However, they believe they can organize their materials adequately, and can inhibit behaviors when necessary.

Informants affirmed the self-reported deficits in behavioral regulation and metacognition, with specific deficits in flexibility, emotional control, and working memory. Informants also reported marked weaknesses in initiating, planning, and self-monitoring. Self- and informant-ratings diverged on only one subscale; informants rated organization of materials as one of the most extreme deficits, but participants did not identify this as an area of weakness.

\section{Self- Versus Informant-Ratings}

The second motivation for this study was to examine self-awareness in $\mathrm{AgCC}$ as represented in the relationship between self and informant BRIEF-A ratings. As predicted, individuals with $\mathrm{AgCC}$ were more likely than the normative sample to report less severe deficits than their informants on the metacognition index, but did not exhibit this effect for behavioral regulation ratings. While this pattern of difference from the normative sample was evident on subscales from both domains (metacognition: initiative, planning, organization of material; behavioral regulation: shifting, and self-monitoring), the general pattern of greater discrepancy on metacognition is consistent with results in traumatic brain injury (Wilson Donders, \& Nguyen, 2011). This finding in AgCC supports the suggestion from Wilson et al. (2011) that the BRIEF MI is particularly sensitive to brain-based deficits in selfawareness, and raises the possibility that MI is specifically sensitive to disruptions in brain connectivity. 


\section{Effects of Participant Characteristics}

Younger participants tended to report greater deficits in behavior regulation than did older participants, but informant-ratings did not vary with participant age. The same pattern was seen in raw scores from the normative sample (Roth et al., 2005, p. 53), resulting in creation of the age-stratified norms used for standardizing our data. However, use of age-stratified norms did not fully account for age-effects on self-reports in AgCC. If we presume, as we have been, that informant-ratings provide a more accurate description than self-ratings, then our findings indicate that deficits in behavioral regulation are not age-dependent in $\mathrm{AgCC}$ (i.e., informant ratings did not correlate with age) and age does not consistently impact our index of self-awareness (i.e., discrepancy between informant- and self-report), but age does impact self-perception of these deficits (i.e., endorsement of impairment declined with age). Relative to informant-reports, younger adults appeared to over-report and older adults underreport deficits in behavior regulation. It is possible that as a result of close contact with parents during early adulthood, younger adults are likely to be receiving stronger and more direct corrective feedback than older adults (who are no longer being "parented"), thus their elevated ratings may reflect awareness of negative feedback more so than self-awareness.

In contrast, participant gender influenced informant- but not self-ratings of metacognition, with informants reporting greater deficits in males than females. This was not the pattern in the normative sample and the norms were not gender-stratified (Roth et al., 2005, p. 53). Once again, if we presume that that informant-ratings provide a more accurate description than self-ratings, our findings indicate that while males with $\mathrm{AgCC}$ exhibit impairments in all aspects of metacognition in daily activities, metacognition is not impaired in females. However, we cannot rule-out the possibility that informants rated males more harshly than females and should examine this in future studies.

\section{Limitations of the Current Study}

Generalizability of the study may be limited by small group size. The study may also be limited by the use of normative data from paper-and-pencil administration, which may differ from online administration in an as yet unknown manner. Online administration also limited experimental control over the testing environment and the ability to monitor compliance with instructions (e.g. to complete the testing independently). Exploration of moderating effects may have been impacted by the use of estimated FSIQ for some participants, and was limited by lack of information about potentially relevant informant characteristics (demographic, social, and psychological). Finally, because this study does not account for objectively measured variations in executive functioning, we are unable to characterize the relationship between specific skills and reports of daily functioning.

\section{Interpretation}

Complex reasoning and problem-solving weakness has been proposed as a core feature of the cognitive profile in AgCC (Brown \& Paul, 2019) and is consistent with executive function deficits in daily life described here. Previously described neuropsychological deficits in this population may inform our understanding of the underlying causes of these daily difficulties. For example, elevated MI ratings are consistent with neuropsychological studies of problem-solving (Brown et al., 2012; Ha, Paul, \& Brown, 2016; Mangum, Paul, 
\& Brown, 2014; Schieffer, 1999), flexibility and cognitive shifting (Marco et al., 2012), and interpretation of second-order meanings in language (Brown, Paul et al., 2005; Paul et al., 2003; Rehmel et al., 2016). Poor in-lab performance on set-shifting and flexibility tasks in $\mathrm{AgCC}$ was largely (but not entirely) explained by diminished processing speed, as well as poor abstract reasoning, both of which should be considered in future studies of set-shifting deficits in everyday life.

Similarly, BRIEF-A ratings indicating poor emotional control in daily life are consistent with previous findings that individuals with AgCC have diminished ability to describe and talk about their emotions and feelings (Brown \& Paul, 2000; Paul et al., 2006; Paul, Pazienza, \& Brown, in press), diminished capacity for reasoning abstractly about complicated socio-emotional situations (Anderson et al, 2017; Young et al., 2019) and making social inferences that involve attribution of mental and emotional states (RenteriaVazquez et al., in press; Paul et al., 2004; Symington et al., 2010; Turk et al., 2010). According to psychological constructionist theories (e.g., Lindquist, MacCormack, \& Shablack, 2015), this weak coupling of language and emotional experiences (with reference to themselves and others) may contribute to poor emotional control.

Finally, discrepant findings on BRIEF-A self- and informant-reports is consistent with previous research reporting naïve self-understanding on personality testing (Brown \& Paul, 2000; Kaplan et al., 2012; Longino, 2011) and with a more recent finding of discordant selfand informant-ratings on a measure of everyday adaptive abilities (Miller, Panos, Brown, \& Paul, 2016). Taken together, self-versus-informant discrepancies in ratings on the BRIEF-A contribute important support for the conclusion that individuals with $\mathrm{AgCC}$ perceive their daily executive and adaptive abilities more favorably than their close relatives, which may reflect poor self-awareness.

Adults with ASD also endorse deficits in everyday executive functioning, and like the adults with AgCC, individuals with ASD tend to rate these deficits as less severe than informants rate them (Johnston et al., 2019). In ASD, these self- or informant-ratings of everyday executive deficits were not associated with cognitive assessment of executive functioning or with ASD symptoms, suggesting that other factors may be negatively impacting these skills in daily life. In contrast with our findings in $\mathrm{AgCC}$, the opposite gender-effect has been reported in adolescents with autism: parents reported greater deficits in females than males who had similar IQ, ASD symptoms, and social/communication skills (White et al., 2017). Future studies comparing daily functioning of adults with $\mathrm{AgCC}$ with and without a diagnosis of ASD, as well as adults with ASD without AgCC may help clarify the underlying challenges shared by and specific to these conditions.

\section{Summary}

Mild to moderate deficiencies in everyday executive functioning were endorsed by individuals with $\mathrm{AgCC}$ themselves, as well as by informants. The deficits are most marked in domains of executive function that involve aspects of behavioral and cognitive flexibility (i.e., shifting and emotional control) and metacognition (i.e., planning, initiating, organizing, working memory). Individuals with $\mathrm{AgCC}$ tend to appraise their own executive functioning problems as less severe than informants, especially with respect to their metacognitive 
functioning. Informants describe greater metacognitive deficits in males with $\mathrm{AgCC}$ than in females. Finally, younger individuals with AgCC tend to rate their deficits more similarly to informants, suggesting that older adults may be less aware of their executive functioning weaknesses.

Brown and Paul (2019) have argued that the core syndrome of AgCC involves reduced cognitive processing speed and deficits in complex reasoning and problem-solving (both aspects of executive functioning). The outcomes of the various subscales of the BRIEF-A provide important support for this understanding of AgCC by demonstrating deficiencies in executive functioning in daily life.

\section{ACKNOWLEDGEMENTS}

Data included in this paper was also utilized in the doctoral dissertations of RM \& JM, at the Fuller Graduate School of Psychology.

\section{FINANCIAL SUPPORT}

This research was not supported by extramural funding.

\section{REFERENCES}

Altman DG, Machin D, Bryant TN, \& Gardner MJ (2000). Statistics with Confidence: Confidence Intervals And Statistical Guidelines, 2nd ed. London: BMJ Books.

Anderson LB, Paul LK, \& Brown WB (2017). Emotional intelligence in agenesis of the corpus callosum. Archives of Clinical Neuropsychology, 32, 267-279. doi: 10.1093/arclin/acx001 [PubMed: 28431033]

Badaruddin DH, Andrews GL, Bölte S, Schilmoeller KJ, Schilmoeller G, Paul LK, \& Brown WS (2007). Social and behavioral problems of children with agenesis of the corpus callosum. Child Psychiatry \& Human Development, 38, 287-302. doi: 10.1007/s10578-007-0065-6 [PubMed: 17564831]

Barr MS \& Corballis MC (2002). The role of the anterior commissure in callosal agenesis. Neuropsychology, 16, 459. doi: 10.1037//0894-4105.16.4.459 [PubMed: 12382985]

Bechara A, Damásio AR, Damásio H, \& Anderson SW (1994). Insensitivity to future consequences following damage to human prefrontal cortex. Cognition, 50(1-3), 7-15. doi: 10.1016/0010-0277(94)90018-3 [PubMed: 8039375]

Bivona U, Ciurli P, Barba C, Onder G, Azcnuda E, Silvestro D, \& Formisano R (2008). Executive function and metacognitive self-awareness after severe traumatic brain injury. Journal of International Neuropsychological Society, 14, 862-868.

Bridgman MW, Brown WS, Spezio ML, Leonard MK, Adolphs R, \& Paul LK (2014). Facial emotion recognition in agenesis of the corpus callosum. Journal of Neurodevelopmental Disorders, 6, 32. doi: 10.1186/1866-1955-6-32 [PubMed: 25705318]

Brown WS, Anderson L, Symington M, \& Paul L (2012). Decision-making in individuals with agenesis of the corpus callosum: Expectancy-valence in the Iowa Gambling Task. Archives of Clinical Neuropsychology: The Official Journal of the National Academy of Neuropsychologists, 27, 532-544. doi: 10.1093/arclin/acs052 [PubMed: 22721927]

Brown WS, Jeeves MA, Dietrich R, \& Burnison DS (1999). Bilateral field advantage and evoked potential interhemispheric transmission in commissurotomy and callosal agenesis. Neuropsychologia, 37, 1165-1180. doi: 10.1016/S0028-3932(99)00011-1 [PubMed: 10509838]

Brown WS, Panos A, \& Paul LK (2020). Attention, impulsivity, and vigilance in agenesis of the Corpus Callosum. Neuropsychology. doi: 10.1037/neu0000685

Brown WS \& Paul LK (2000). Cognitive and psychosocial deficits in agenesis of the corpus callosum with normal intelligence. Cognitive Neuropsychiatry, 5, 135-157. doi: 10.1080/135468000395781 
Brown WS \& Paul LK (2019). The neuropsychological syndrome of agenesis of the corpus callosum. Journal of the International Neuropsychological Society. 25, 324-330. doi: 10.1017/ S135561771800111X [PubMed: 30691545]

Brown WS, Paul LK, Symington M, \& Dietrich R (2005). Comprehension of humor in primary agenesis of the corpus callosum. Neuropsychologia, 43, 906-916. doi: 10.1016/ j.neuropsychologia.2004.09.008 [PubMed: 15716161]

Brown WS, Symington M, VanLancker-Sidtis D, Dietrich R, \& Paul LK (2005). Paralinguistic processing in children with callosal agenesis: Emergence of neurolinguistic deficits. Brain and Language, 93, 135-139. doi: 10.1016/j.bandl.2004.09.003 [PubMed: 15781301]

Brown WS, Thrasher ED, \& Paul LK (2001). Interhemispheric Stroop effects in partial and complete agenesis of the corpus callosum. Journal of the International Neuropsychological Society, 7, 302311. doi: 10.1017/S1355617701733048 [PubMed: 11311031]

Butcher JN, Graham JR, Ben Porath YS, Tellegen A, Dahlstrom WG, \& Kaemmer B (2001). Manual for Administration, Scoring, and Administration (revised ed.). Minneapolis, MN: University of Minnesota Press.

Campbell I (2007). Chi-squared and Fisher-Irwin tests of two-by-two tables with small sample recommendations. Statistics in Medicine 26, 3661-3675. [PubMed: 17315184]

Chiarello C (1980). A house divided? Cognitive functioning with callosal agenesis. Brain and Language, 11, 128-158. doi: 10.1016/0093-934X(80)90116-9 [PubMed: 7427714]

Erickson RL, Young CY, Paul LK, \& Brown WS (2013). WAIS-III index scores in individuals with agenesis of the corpus callosum. Final Program Forty First Annual Meeting International Neuropsychological Society February 6-9, 2013 Waikoloa, Hawaii, USA. (2013). Journal of the International Neuropsychological Society, 19(S1), 224. doi: 10.1017/S1355617713000362

Erickson RL, Paul LK, Brown WS (2014). Verbal learning and memory in agenesis of the corpus callosum. Neuropsychologia, 60, 121-130. doi: 10.1016/j.neuropsychologia.2014.06.003 [PubMed: 24933663]

Fischer M, Ryan SB, \& Dobyns WB (1992). Mechanisms of interhemispheric transfer and patterns of cognitive function in acallosal patients of normal intelligence. Archives of Neurology, 49, 271277. doi: 10.1001/archneur.1992.00530270085023 [PubMed: 1536630]

Glass HC, Shaw GM, Ma C, \& Sherr EH (2008). Agenesis of the corpus callosum in California 1983-2003: A population-based study. American Journal of Medical Genetics, 146A, 2495-2500. doi: 10.1002/ajmg.a.32418 [PubMed: 18642362]

Gott PS \& Saul RE (1978). Agenesis of the corpus callosum: Limits of functional compensation. Neurology, 28, 1271-1279. doi: 10.1212/WNL.28.12.1272

Ha J, Paul LK, \& Brown WS (2016). Verbal problem solving in individuals with agenesis of the corpus callosum. Meeting Proceedings, Forty Fourth Annual Meeting International Neuropsychological Society February 3-6, 2016 Boston, Massachusetts, USA. (2016). Journal of the International Neuropsychological Society, 22(S1), 4. doi: 10.1017/S1355617716000734

Imamura T, Yamadori A, Shiga Y, Sahara M, \& Abiko H (1994). Is disturbed transfer of learning in callosal agenesis due to a disconnection syndrome? Behavioral Neurology, 7, 43-48. doi: 10.3233/ BEN-1994-7201 [PubMed: 24487287]

Jeeves MA (1979). Some limits to interhemispheric integration in cases of callosal agenesis and partial commissurotomy. In Steele Russell I, van Hof MW, \& Berluchhi G (Eds.), Structure and Function of Cerebral Commissures (pp. 449-474). London: Macmillan.

Jinkins J, Whittemore A, \& Bradley W (1989). MR imaging of callosal and corticocallosal dysgenesis. American Journal of Neuroradiology, 10, 339-344. [PubMed: 2494854]

Johnston K, Murray K, Spain D, Walker I, \& Russell A (2019). Executive function: Cognition and behaviour in adults with autism spectrum disorders (ASD). Journal of Autism and Developmental Disorders, 49, 4181-4192. doi: 10.1007/s10803-019-04133-7 [PubMed: 31281952]

Kaplan JM, Brown WS, Adolphs R, \& Paul LK (2012). Psychological profile in agenesis of the corpus callosum [Abstract]. Poster presented at the Society for Neuroscience Annual Meeting: October 13-17, 2012 New Orleans, LA, USA. 198.19.

J Int Neuropsychol Soc. Author manuscript; available in PMC 2022 January 10. 
Karnath HO, Schumacher M, \& Wallesch CW (1991). Limitations of interhemispheric extracallosal transfer of visual information in callosal agenesis. Cortex, 27, 345-350. doi: 10.1016/ S0010-9452(13)80141-4 [PubMed: 1879164]

Lau YC, Hinkley LB, Bukshpun P, Strominger ZA, Wakahiro ML, Baron-Cohen S, ... Marco EJ 2013. Autism traits in individuals with agenesis of the corpus callosum. Journal of Autism Developmental Disorders, 43, 1106-1118. doi: 10.1007/s10803-012-1653-2. [PubMed: 23054201]

Lindquist KA, MacCormack JK, \& Shablack H (2015). The role of language in emotion: predictions from psychological constructionism. Frontiers in Psychology, 6, 444. doi: 10.3389/ fpsyg.2015.00444 [PubMed: 25926809]

Longino SM (2011). Analysis of content scales of the Minnesota Multiphasic Personality Inventory-2 in complete and partial agenesis of the corpus callosum. Dissertation Abstracts International, 73, 1304.

Mangum R, Paul LK, \& Brown WS (2014). Twenty questions problem-solving and strategy in individuals with agenesis of the corpus callosum. Final Program Forty Second Annual Meeting International Neuropsychological Society February 12-15, 2014 Seattle, Washington, USA. (2014). Journal of the International Neuropsychological Society, 20(S1), 260. doi: 10.1017/ S1355617714000381

Marco EJ, Harrell KM, Brown WS, Hill SS, Jeremy RJ, Kramer JH, ... Paul LK (2012). Processing speed delays contribute to executive function deficits in individuals with agenesis of the corpus callosum. Journal of the International Neuropsychological Society, 18, 521-529. [PubMed: 22390821]

Miller JS, Brown WS, Mangum R, \& Paul LK (2020). Adaptive behavior in AgCC: Self and informant reports. Manuscript submitted for publication.

Miller JS, Panos AH, Brown WS \& Paul LK (2016). Adaptive skills in high-functioning adults with ACC and ASD. Meeting Proceedings, Forty Fourth Annual Meeting International Neuropsychological Society February 3-6, 2016 Boston, Massachusetts, USA. Journal of the International Neuropsychological Society, 22(S1), 5. doi: 10.1017/S1355617716000734

Paul LK, Brown WS, Adolphs R, Tyszka J, Richards LJ, Mukherjee P, \& Sherr EH (2007). Agenesis of the corpus callosum: Genetic, developmental and functional aspects of connectivity. Nature Reviews Neuroscience, 8, 287-299. doi: 10.1038/nrn2107 [PubMed: 17375041]

Paul LK, Corsello C, Kennedy D, \& Adolphs R (2014). Agenesis of the corpus callosum and autism: A comprehensive comparison. Brain, 137, 1813-1829. [PubMed: 24771497]

Paul LK, Erickson RL, Hartman JA, \& Brown WS (2016). Learning and memory in individuals with agenesis of the corpus callosum. Neuropsychologia, 86, 183-192. doi: 10.1016/ j.neuropsychologia.2016.04.013 [PubMed: 27091586]

Paul LK, Lautzenhiser A, Brown WS, Hart A, Neumann D, Spezio M, \& Adolphs R (2006). Emotional arousal in agenesis of the corpus callosum. International Journal of Psychophysiology, 61, 47-56. doi: 10.1016/j.ijpsycho.2005.10.017 [PubMed: 16759726]

Paul LK, Pazienza S, \& Brown WS (in press). Alexithymia and somatization in agenesis of the corpus callosum. Social Cognitive and Affective Neuroscience.

Paul LK, Schieffer B, \& Brown WS (2004). Social processing deficits in agenesis of the corpus callosum: Narratives from the Thematic Apperception Test. Archives of Clinical Neuropsychology, 19, 215-255. doi: 10/1016/S0887-6177(03)00024-6 [PubMed: 15010087]

Paul LK, VanLancker-Sidtis D, Schieffer B, \& Brown WS (2003). Communicative deficits in individuals with agenesis of the corpus callosum: Nonliteral language and affective prosody. Brain and Language, 85, 313-324. doi: 10.1016/S0093-934X(03)00062-2 [PubMed: 12735947]

Rehmel JL, Brown WS, \& Paul LK (2016). Proverb comprehension in individuals with agenesis of the corpus callosum. Brain \& Language, 160, 21-29. doi: 10.1016/j.bandl.2016.07.001 [PubMed: 27448531]

Renteria-Vazquez T, Brown WS, Kang C, Graves M, Castelli F, \& Paul LK (in press). Social inferences in agenesis of the corpus callosum and autism: Semantic analysis and topic modeling. Journal of Autism and Developmental Disorders.

Richardson JTE (2011). The analysis of $2 \times 2$ contingency tables - Yet again. Statistics in Medicine, 30, 890. [PubMed: 21432882] 
Roth RM, Isquith PK, \& Gioia GA (2005). Behavioral Rating Inventory of Executive Function-Adult Version. Lutz, FL: Psychological Assessment Resources.

Sauerwein HC \& Lassonde M (1983). Intra-and interhemispheric processing of visual information in callosal agenesis. Neuropsychologia, 21, 167-171. doi: 10.1016/0028-3932(83)90084-2 [PubMed: 6866259]

Sauerwein HC \& Lassonde M (1994). Cognitive and sensori-motor functioning in the absence of the corpus callosum: Neuropsychological studies in callosal agenesis and callosotomized patients. Behavioral Brain Research, 64, 229-240. doi: 10.1016/0166-4328(94)90135-X

Schieffer B, Paul L, \& Brown W (2000). Deficits in complex concept formation in agenesis of the corpus callosum [Abstract]. Journal of the International Neuropsychological Society, 6, 164.

Schieffer BM (1999). Concept formation, problem-solving and memory encoding abilities in individuals with congenital agenesis of the corpus callosum and normal intelligence. Dissertation Abstracts International, 62(3), 1597.

Siffredi V, Anderson V, Leventer RJ, \& Spencer-Smith MM (2013). Neuropsychological profile of agenesis of the corpus callosum: A systematic review. Developmental Neuropsychology, 38, 3657. [PubMed: 23311314]

Smith LA \& Rourke BP (1995). Callosal agenesis. In Rourke BP (Ed.), Syndrome of nonverbal learning disabilities: Neurodevelopmental manifestations (pp. 45-92). New York: Guilford Press.

Solursh LP, Margulies AI, Ashem B, \& Stasiak EA (1965). The relationships of agenesis of the corpus callosum to perception and learning. Journal of Nervous and Mental Disease, 141, 180-189. doi: 10.1097/00005053-196508000-00005

Sperry RW (1974). Lateral specialization in the surgically separated hemispheres. In Schmitt FO \& Worden PG (Eds.), Neurosciences: Third study program. Cambridge, MA: MIT Press.

Strauss E, Sherman EMS, \& Spreen O (2006). A Compendium of Neuropsychological Tests: Administration, Norms, and Commentary, (3rd ed.). New York, NY: Oxford University Press.

Symington SH, Paul LK, Symington MF, Ono M, \& Brown WS (2010). Social cognition in individuals with agenesis of the corpus callosum. Social Neuroscience, 5, 296-308. doi: 10.1080/17470910903462 [PubMed: 20162492]

Turk AA, Brown WS, Symington M, \& Paul LK (2010). Social narratives in agenesis of the corpus callosum: Linguistic analysis of the Thematic Apperception Test. Neuropsychologia, 48, 43-50. doi: 10.1016/j.neuropsychologia.2009.08.009 [PubMed: 19686767]

Verhoeven E, Marijnissen N, Berger H, Oudshoorn J, Sijde A, \& Teunisse J (2012). Brief report: Relationship between self-awareness of real-world behavior and treatment outcome in autism spectrum disorders. Journal of Autism \& Developmental Disorders, 42(5), 889-894. [PubMed: 21698498]

Wechsler D (1997). WAIS-III: Wechsler Adult Intelligence Scale (3rd ed.). San Antonio, TX: The Psychological Corporation.

Wechsler D (2001). Wechsler Test of Adult Reading. San Antonio, TX: Pearson.

Wechsler D (2003). Wechsler Intelligence Scale for Children, (4th ed.). London: Pearson.

Wechsler D (2005). Wechsler Individual Achievement Test (WIAT II), (2nd ed.). London: The Psychological Corp.

White EI, Wallace GL, Bascom J, Armour AC, Register-Brown K, Popal HS, ... Kenworthy L (2017). Sex differences in parent-reported executive functioning and adaptive behavior in children and young adults with autism spectrum disorder. Autism Research: Official Journal of the International Society for Autism Research, 10(10), 1653-1662. doi: 10.1002/aur.1811 [PubMed: 28568910]

Wilson KR, Donders J, \& Nguyen L (2011). Self and parent ratings of executive functioning after adolescent traumatic brain injury. Rehabilitation Psychology, 56(2), 100-106. [PubMed: 21574728]

Young CM, Folsom RC, Paul LK, Su J, Mangum RW, \& Brown WS (2019). Awareness of consequences in agenesis of the corpus callosum: Semantic analysis of responses. Neuropsychology, 33, 275-284. doi: 10.1037/neu0000512 [PubMed: 30667251] 


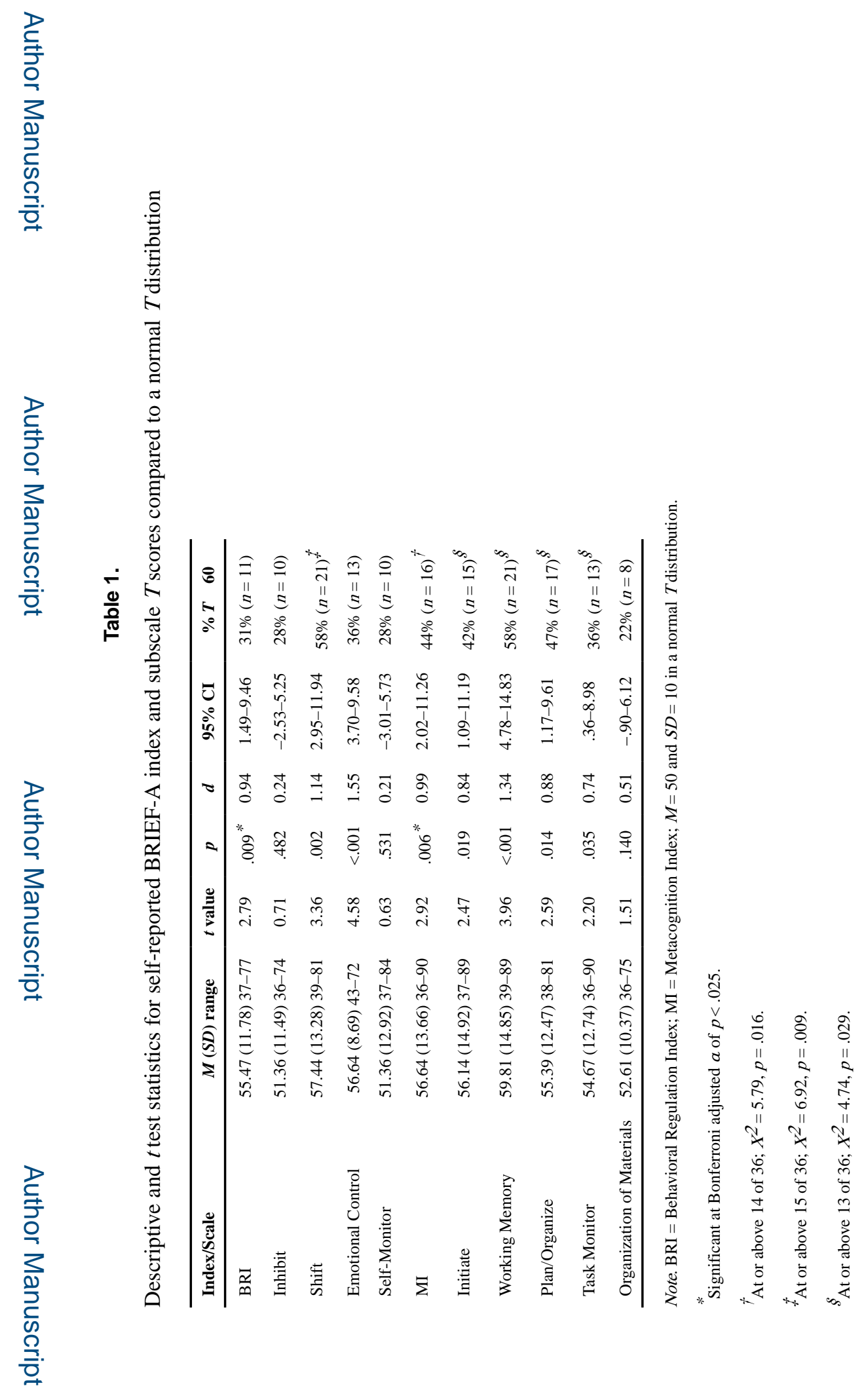

J Int Neuropsychol Soc. Author manuscript; available in PMC 2022 January 10. 


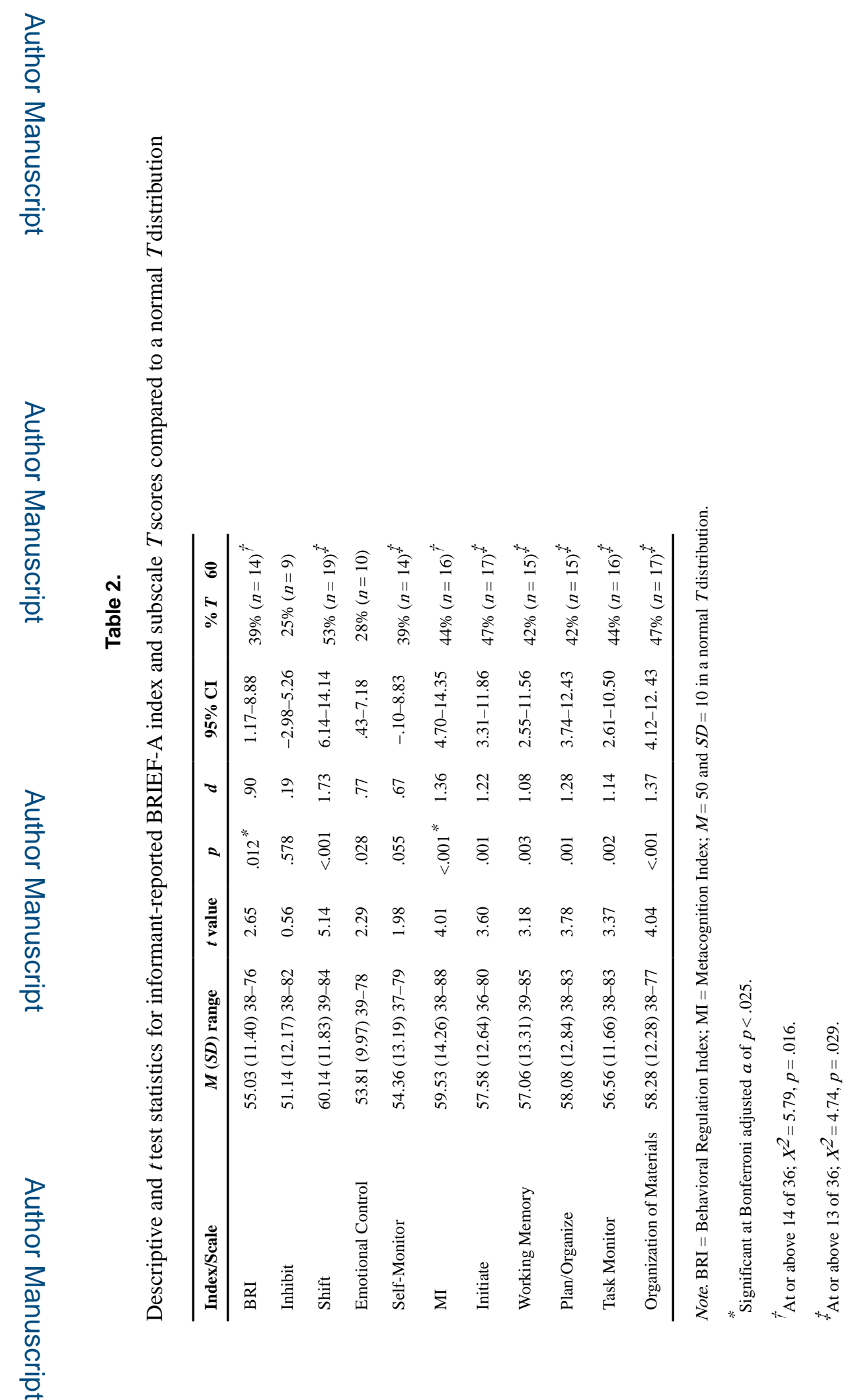

J Int Neuropsychol Soc. Author manuscript; available in PMC 2022 January 10. 


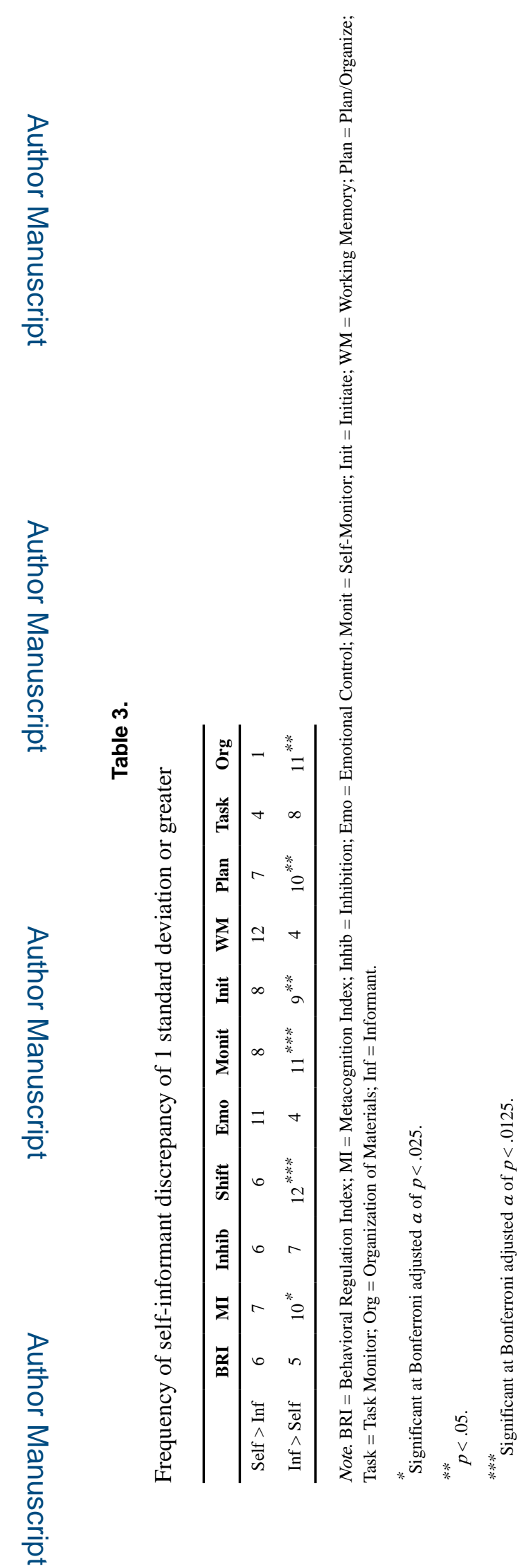

J Int Neuropsychol Soc. Author manuscript; available in PMC 2022 January 10. 